discrimination and calibration for performance of a model. Framingham risk score (FRS) for cardiovascular disease is a widely used one which has been validated in different countries but its clinical usefulness has been neglected.

Methods We checked discrimination of FRS and so its calibration and clinical usefulness before and after recalibration in a population based cohort, Tehran lipid and glucose study, of 2640 men and 3584 women aged $30-74$ years. To check clinical usefulness, we used decision curve analysis (DCA) and calculated net benefit of treatment for patients with $\geq 20 \%$ of 10 year probability of disease according to FRS model.

Results The area under the curve for FRS model, was 0.794 and 0.838 for men and women respectively. The original model had a poor calibration but got a good one after recalibration (HosmerLemeshow $\chi^{2}$ statistic of 16.8 for men and 18.4 for women). Based on DCA, FRS was clinically useful in cut points of $10 \%-30 \%$, as threshold probability of disease that a patient should be treated, before and after recalibration. The net benefit of model to treat patients at cut point of $20 \%$ did not differ significantly before and after recalibration in both men and women ( $p>0.3$ based on bootstrap resampling).

Conclusion Original FRS has a good discrimination and poor calibration in Iran but considering clinical usefulness, it can be used even without recalibration.

\section{P1-32 TESTING LIFE COURSE MODELS TO INVESTIGATE THE EFFECT OF SOCIOECONOMIC POSITION ON CRYSTALLISED COGNITIVE FUNCTION IN OLDER AGE, ACCOUNTING FOR MISSING DATA}

doi:10.1136/jech.2011.142976c.26

\begin{abstract}
${ }^{1,2} \mathrm{R}$ Landy, ${ }^{1} \mathrm{~J} \mathrm{Head},{ }^{2} \mathrm{M}$ Richards, ${ }^{2} \mathrm{R}$ Hardy. ${ }^{*}$ Department of Epidemiology and Public Health, University College London, London, UK; ${ }^{2}$ MRC Unit for Lifelong Health and Ageing, Department of Epidemiology and Public Health, London, UK
\end{abstract}

Objective To investigate how lifetime socioeconomic position (SEP) is associated with later-life crystallised cognitive function, accounting for different missing data mechanisms.

Participants A nationally representative population sample born in 1946 (MRC National Survey of Health and Development; NSHD, $\mathrm{N}=5362$ ), and a sample of British civil servants (Whitehall II; WHII, $\mathrm{N}=10308$ ).

Methods Novel structured statistical approach to distinguish between accumulation and sensitive period life course models using SEP measures from childhood, early-adulthood and midlife. Results of complete case (CC) (assuming missing completely at random), multiple imputation (MI) (missing at random) and a Heckman selection model (missing not at random) were compared.

Outcomes National Adult Reading Test, age 53 (NSHD); Mill Hill Test, age 55-79 (WHII)

Results NSHD: After adjusting for childhood cognitive function, the best fitting model was an accumulation model allowing SEP at each time point to have its own estimate. However estimates varied by missing data method (women: childhood SEP: CC: coefficient $=1.11$ (95\% CI 0.15 to 2.06 ), MI: coefficient $=1.82$ (95\% CI 0.87 to 2.76 ), Heckman: coefficient $=0.70$ (95\% CI -0.38 to 1.78$)$ ). WHII (not adjusted for childhood cognition): the best fitting model represented accumulation in adulthood only, with childhood SEP not significant. Conclusion Despite adjustment for childhood cognitive score, childhood SEP remains important in NSHD, whereas in Whitehall II childhood SEP was not associated with cognitive function. These differences may be due to recall bias of early SEP in WHII. Our findings demonstrate the utility of the method for distinguishing models of how SEP across the life course influences cognition and the importance of dealing with missing data.

\section{P1-33 ADULT DNA METHYLATION IN RELATION TO PRENATAL NUTRITION AND RISK FACTORS FOR CARDIO-VASCULAR DISEASE}

doi:10.1136/jech.2011.142976c.27

L H Lumey, ${ }^{*}$ M B Terry, L Delgado-Cruzata, K Gonzales, 0 Wang, Y Liao, E Susser, I McKeague, R Santella. Columbia University, New York, USA

Epigenetic changes may play an important role in the development of adult disease. Exposure to a prenatal famine environment has been associated with a persistent decrease in DNA methylation of the IGF2 gene, and other early life factors like birthweight with adult genomic DNA methylation. We evaluated genomic DNA methylation in relation to prenatal nutrition and CVD risk factors. Our study includes 353 births from three clinics with prenatal exposure to the Dutch famine of 1944-1945, 296 before-or-after the famine births as unexposed time controls, and 311 same-sex siblings of either group as unexposed family controls. All study subjects underwent medical examinations and DNA collections at a mean age of $\sim 58$ years. We used Luminometric methylation (LUMA) and LINE-1 (Long Interspersed Nucleotide Element 1) pyrosequencing assays to quantify genomic DNA methylation. Mean DNA methylation by LUMA was 75.5\% (SD 2.2) and by LINE-1 77.1\% (SD 2.5). Neither was associated with prenatal famine exposure. Using all controls, famine exposure showed a decline of $0.12 \%(95 \% \mathrm{CI}$ -0.42 to $0.18 ; p=0.44)$ by LUMA and an increase of $0.05 \%(-0.33$ to $0.21 ; \mathrm{p}=0.68)$ by LINE- 1 . Using family controls, the increases were $0.36 \%$ (LUMA) and $0.16 \%$ (LINE-1). Neither assay was associated with adult cholesterol, blood pressure, body size, type 2 diabetes mellitus, gender or age. Our results suggest that these genomic DNA methylation markers may not be associated with prenatal famine; further work should target regions in the genome that may be differentially methylated in response to early life exposures.

\section{P1-34 CAN ASSOCIATIONS BETWEEN PRENATAL NUTRITION AND BODY MASS INDEX BE EXPLAINED BY COMIMON GENETIC VARIANTS? FINDINGS FROM THE DUTCH HUNGER WINTER FAMILIES STUDY}

doi:10.1136/jech.2011.142976c.28

${ }^{1}$ L H Lumey, ${ }^{* 2} \mathrm{~A}$ Vaarhorst, ${ }^{3} \mathrm{~A} D$ Stein, ${ }^{2} \mathrm{~B}$ Heijmans. ${ }^{1}$ Columbia University, New York, USA; ${ }^{2}$ Leiden Univ Med Ctr, Leiden, The Netherlands; ${ }^{3}$ Emory University, Atlanta, Georgia, USA

Genome wide association (GWAS) studies have identified single nucleotide polymorphisms (SNPs) related to body mass index (BMI $\mathrm{kg} / \mathrm{m}^{2}$ ). Associations have also been reported between fetal malnutrition and BMI. We use the circumstances of the Dutch Hunger Winter of 1944-1945 to further examine these relations. We studied 348 adult men and women born in affected cities in the western Netherlands who had been exposed to famine during pregnancy, 294 born before or after the famine as time-controls, and 305 same-sex unexposed siblings of above groups as family controls. Mean age at examination was 58 years. We evaluated common variants in the FTO, TMEM18, MC4R, GNPDA2, BDNF, SEC16B, NEGR1, SH2B1, SFRS10, MTCH2, and KCTD15 genes related to BMI. A genetic risk score was calculated for each individual by summing the number of risk alleles in these genes. Scores were also weighted using recent GWAS estimates of gene specific changes in BMI per risk allele. Institutional ethics committees gave the appropriate approvals for the study. Genetic risk scores had a mean of 11.4 (SD 2.2) and were not related to prenatal famine. Adult BMI was 1.34 units higher among famine exposed (95\% CI 0.73 to 1.94 ) and further adjustments for risk score had little effect. There was no statistical 
interaction between famine exposure and genetic risk scores with regard to BMI outcomes. Analyses with weighted risk scores confirm these patterns. Common genetic variants related to BMI do not explain the association between prenatal famine and adult BMI in our study population.

\section{P1-35 ON THE USE OF EMPIRICAL LIKELIHOOD BASED METHODS TO ACHIEVE BALANCE ON MEASURED CONFOUNDERS}

doi:10.1136/jech.2011.142976c.29

G Luta, ${ }^{*}$ A Dragomir, A Barbo, C Loffredo. Georgetown University, Washington, DC, USA

Introduction One of the limitations of the statistical methods that use propensity scores, such as those involving adjustment for the propensity score, matching, subclassification, and inverse probability of treatment weighting, is that they do not achieve exact balance with respect to the measured confounders. Empirical likelihood is a nonparametric method with desirable statistical properties that is perfectly suited to perform the reweighting of the data as to achieve exact balance on measured confounders.

Methods We describe statistical methods that use empirical likelihood to construct weights that add up to one and produce exact balance when applied to the data. For the case involving only categorical confounders, the empirical likelihood based methods produce weights similar to those generated by the inverse probability weighting or standardisation methods. The new methods can handle both categorical and continuous confounders in a unified manner, and allow the incorporation of balancing constraints ranging from simple equalities of means/proportions to more complex constraints related to the comparison of distributions.

Results Under different scenarios of interest, we perform simulations to compare the statistical properties of the proposed method with the inverse probability weighting method. For comparative purposes we also use both methods to evaluate the association between cardiac malformations and birthweight using data from the Washington-Baltimore Infant Study.

Conclusion The proposed empirical likelihood based method performs well and should be used as complementary to the currently available propensity score based methods.

\section{P1-36 DATA SOURCES ON DRUG SAFETY EVALUATION: A REVIEW OF RECENT PUBLISHED META-ANALYSIS}

doi:10.1136/jech.2011.142976c.30

${ }^{1,2} \mathrm{C}$ Alves, ${ }^{1,3} \mathrm{~F}$ Batel-Marques, ${ }^{1,3} \mathrm{~N}$ Craveiro, ${ }^{2,3} \mathrm{~A}$ F Macedo. ${ }^{*}$ School of Pharmacy, University of Coimbra, Coimbra, Portugal; ${ }^{2}$ Health Sciences Research Centre, University of Beira Interior, Covilhã, Portugal; ${ }^{3}$ Central Portugal Regional Pharmacovigilance Centre, AIBILI, Coimbra, Portugal

Introduction Harmful effects of medicines should be reviewed with similar rigour as therapeutic benefits. Most evidence on harms is obtained from post marketing surveillance, so the use of metaanalysis to pool safety information presents challenges of inherent biases and differences in study designs. Yet, it's crucial to provide an accurate safety profile of pharmacological interventions. We aimed to describe the data sources of published meta-analysis of adverse drug effects.

Method We searched meta-analysis published in the last 5 years in six medical journals with the highest impact factor. All the metaanalysis focussing primarily on adverse effects of pharmacological interventions, with pooled results, were included and the characteristics assessed.

Results A total of 61 meta-analysis were included, 16 were published in JAMA, 13 in Lancet, 11 in BMJ, 10 in Ann Intern Med, nine in Arch Intern Med and two in NEJM. Of these $90.2 \%(n=55)$ included only experimental studies, two included only observational studies and four meta-analysis comprised both type of studies. Less than half $(47.5 \% ; n=29)$ of the meta-analysis assessed the quality of the included studies according to specified recommendation statements, and only $18(29.5 \%)$ considered unpublished studies.

Conclusion The majority of meta-analysis of adverse drug effects included only experimental studies, less than half assessed their quality and few considered unpublished studies. These results reinforce the need for methodological research to clarify the role of meta-analysis in Pharmacovigilance and evaluate how to pool safety information from different surveillance methods, to provide an accurate safety profile of pharmacological interventions.

\section{P1-37 WITHDRAWN}

\section{P1-38 MODELLING THE FUTURE BURDENS OF CHRONIC DISEASE THE LESSONS FROM FORESIGHT AND BEYOND}

doi:10.1136/jech.2011.142976c.31

${ }^{1} \mathrm{~T}$ Marsh, ${ }^{*}{ }^{2} \mathrm{~K}$ McPherson, ${ }^{1} \mathrm{M}$ Brown, ${ }^{1} \mathrm{~K}$ Rtveladze. ${ }^{1}$ National Heart Forum, London, UK; ${ }^{2}$ University of Oxford, Oxford, UK

Introduction As the prevalence of chronic diseases continues to climb, the challenges of quantifying the impact of this epidemic to inform decision makers becomes more urgent. Drawing on experiences of work in England, USA, Brazil, Mexico and Russian Federation we will demonstrate how the application of micro simulation modelling can lead to a systematic understanding of the associated morbidities, economic burden and inform policy makers form effective strategies and build the political will for change.

Method The application of micro-simulation modelling techniques to understand the future impact of changes in trends in tobacco consumption and obesity rates and the potential impact of policy interventions.

Results The work initially undertaken for the Foresight Tackling Obesities research was instrumental in galvanising a cross government strategy in England, Healthy Weight, Healthy Lives, subsequent outputs from the simulations in the USA, Brazil, Mexico and Russian Federation should also inform policy in those countries.

Conclusion Morbidity and the economic burden of chronic disease is a practical metric for comparative assessment of health risks, as exemplified by its use by international organizations such as the World Bank, the WHO and the Organisation Economic Co-operation and Development. Nonetheless, the applications for simulation models of morbidity consequences of chronic disease can well go beyond projecting the growth of the problem to the society. A modelling framework provides a useful infrastructure for the comparative evaluation of the effectiveness and return-on-investment of potential policies aimed to alter the drivers and determinants of obesity epidemic.

\section{P1-39 IN RANDOMISATION WE TRUST? RESEARCH REACTIVITY PRODUCES BIAS IN BEHAVIOUR CHANGE TRIALS}

doi:10.1136/jech.2011.142976c.32

\author{
${ }^{1} \mathrm{~J}$ McCambridge, ${ }^{*}{ }^{2} \mathrm{~K}$ Kypri, ${ }^{1} \mathrm{D}$ Elbourne. ${ }^{1}$ LSHTM, London, UK; ${ }^{2}$ University of \\ Newcastle, Newcastle, New South Wales, Australia
}

Introduction Behaviour change trials are increasingly important in public health. Although there has been longstanding awareness of pre-test sensitisation and the Hawthorne effect, the implications of participant reactivity in behaviour change trials are largely unstudied. The aim here is to explore the mechanisms by which biases stemming from the unintended consequences of research participation may be introduced in trials. 